\title{
On the submarine communication cable JASC ground self-potential stability
}

\author{
Sergey S. Starjinsky and Valerian M. Nikiforov \\ V. I. Il'ichev Pacific Oceanological Institute Far Eastern Branch Russian Academy of Sciences, 43 Baltiyskaya Str., \\ Vladivostok, 690041, Russia
}

(Received January 25, 2005; Revised June 23, 2005; Accepted June 27, 2005)

\begin{abstract}
This investigation was undertaken for searching the source of linear trends in JASC submarine cable data over the time interval of 6 years and estimating the electrode noise level. One of the reasons for a trend is probably the potential instability in the cable ground, which happened because the titanium electrodes of the ground were placed in sea water that is electrochemically active. To study this phenomenon, we have registered the voltage variations of three titanium JASC cable electrodes, which were closely spaced $(\sim 15 \mathrm{~cm})$ and placed at a depth of $\sim 15 \mathrm{~m}$ near the coast for 70 days, synchronously with JASC cable voltage observations. The electrode noise level and coherence between channels were estimated and linear trends in electrode pair voltage variation were revealed. The estimated linear trend magnitude is about $0.28 \mathrm{mV} /$ day, which is comparable with $0.21 \mathrm{mV} / \mathrm{day}$ earlier observed in JASC submarine cable data. We concluded that the linear trend in JASC cable data is, at least partly, probably caused by the titanium electrodes potential variations. This linear trend should be registered simultaneously with JASC cable voltage observation, keeping in mind the possibility of finding a procedure for removing it, in order to enhance the signal/noise ratio in data.
\end{abstract}

Key words: Submarine cable, electrode potential, coherence function, amplitude spectra, linear trend.

\section{Introduction}

The voltage measured at a long submarine Japan sea cable (JASC) is caused by three principal types of sources: geomagnetic field fluctuations, the dynamo interaction of moving conductive sea water with a steady magnetic field, and the electromagnetic processes in the Earth's core. The first two sources are characterized by the largest amplitudes in a wide frequency range (Chave et al., 1992; Lanzerotti et al., 1986; Medford et al., 1989; Vanyan et al., 1998), while the third one is small and to be observed at a very long period of about ten or more years (Shimizu and Utada, 2003). These variations will have a linear trend over a comparatively short time interval. The same occurrence will be true for any other submarine cable. The data for the linear drifts in different submarine cables' voltage variation were observed and analyzed for approximately four years by Shimizu et al. (1998) and linear trends of the order of $10^{-4}-10^{-5} \mathrm{mV} / \mathrm{km} \cdot$ day were found. The most important aim of submarine cables is to detect the response in the electric field caused by the Earth's core dynamics. It is clear that to study a low level signals with a low signal to noise ratio $(\mathrm{S} / \mathrm{N})$, we need to estimate a certain level of noise in the obtained data. One of the inherent noise sources is the electrode potential instability. As a rule, in sea magnetotelluric investigation, the well studied low noise $\mathrm{Ag}-\mathrm{AgCl}$ electrodes and other unpolarized electrodes are used (Webb et al., 1985; Constable et al., 1998; Perrier et al., 1997). The long-term drifts of some types of electrodes have been stud-

Copyright (C) The Society of Geomagnetism and Earth, Planetary and Space Sciences (SGEPSS); The Seismological Society of Japan; The Volcanological Society of Japan; The Geodetic Society of Japan; The Japanese Society for Planetary Sciences; TERRAPUB. ied in the laboratory and in the field (Perrier et al., 1997). As a result of this study, linear trends were revealed (the authors believe that one cause of this process is because of that irreversible nature). Some types of metallic electrodes placed in soil were also studied in the laboratory (Petiau and Dupis, 1980). Stainless steel electrodes were used for the streaming potential measurement of rock samples (Lorne $e t$ al., 1999) and it was found that electrode instabilities could be corrected. Egbert et al. (1992) discussed the lead electrode instabilities in regards to the impedance estimation at very low frequencies. In-situ investigations of metallic electrode potential variation are unknown to the authors. This is the reason why we have made an attempt to estimate the submarine cable JASC ground potential stability using seaground design features.

\section{Electrode Potential Variation Measurement}

The JASC cable grounds were placed at the geographical coordinates $37^{\circ} 40.2^{\prime} \mathrm{N}$ and $137^{\circ} 58.8^{\prime} \mathrm{E}$ at a depth of $1.5 \mathrm{~km}$ near the shore line of Japan and $42^{\circ} 48^{\prime} \mathrm{N}$ and $132^{\circ} 49^{\prime} \mathrm{E}$ at an approximate depth of $15 \mathrm{~m}$ and a distance of $0.5 \mathrm{~km}$ away from the Russian shore (Vanyan et al., 1998). The distance between the electrodes is $700 \mathrm{~km}$ or so. At the Russian end of the JASC cable, the sea electrode which consists of four titanium rods of $\sim 0.15 \mathrm{~m}$ long and $0.015 \mathrm{~m}$ in diameter with $\sim 0.15 \mathrm{~m}$ spacing, was installed in 1969 at the present position. The rods are fixed to an isolated material bar (epoxy resin) and set firmly on a concrete frame of $0.8 \mathrm{~m} \times 0.8 \mathrm{~m}$ in size (see Fig. 1). The housing is closed from the top and from the bottom by concrete covers, and there are four holes in the frame to allow sea water to circulate. Each rod is connected to the isolated copper thread of the cable which is $2 \mathrm{~mm}$ in diameter. The cable, 
Nakhodka costal station

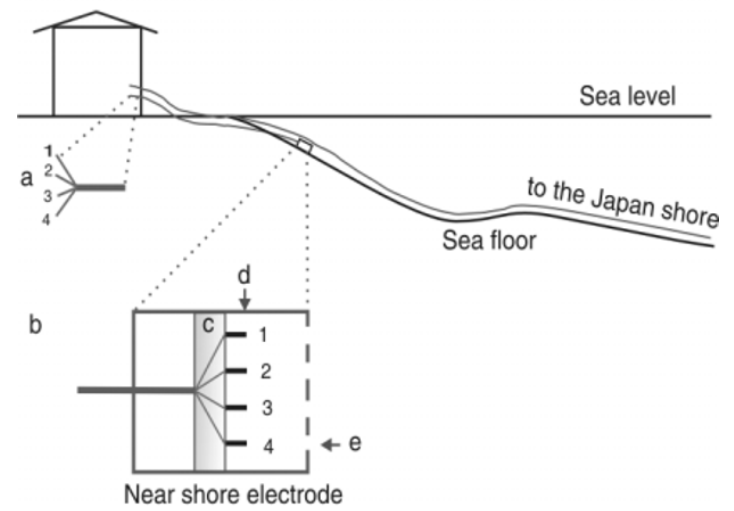

Fig. 1. (A) The cable output at Nakhodka station and (B) the near-shore electrode construction; insulation material bar (c), concrete frame (d) and sea water inlet holes (e).

placed between sea-ground and station, is approximately 5 $\mathrm{km}$ long. The ends of these threads have been shortened at the Nakhodka station and connected to a digital multimeter 7561 (input resistance $>10^{9} \mathrm{Ohm}$ ) for permanent voltage registration.

It is well known that the electric potential resulting from the evolution of the electrode itself or from its interface with the sea water can be identified when the potential differences between the closely spaced electrodes are compared (Webb et al., 1985). In order to organize the rods for measurement, we disconnected the shortened threads, reserving one rod to provide permanent JASC long-term data registration. The other three rods were used for testing the electrode stability; they were connected to two channels (the second and the third) of the 24-bit analog-to-digital converter (ADC) with four differential channels operated by a driver program on a notebook computer. One of the three rods was common for both channels 2 and 3 . We used a commercially available ADC type E-24, which has an input impedance of $4 \times 10^{6} \mathrm{Ohm}$, dynamic range of $\pm 2.5 \mathrm{~V}$, and frequency range from $10^{3} \mathrm{~Hz}$ to $\mathrm{DC}$. The first ADC channel was connected to the first rod and to the electrode near the Japanese coast, that is parallel to a permanent JASC cable voltage registration. The apparatus functioned from 12 March to 21 May, 2003 at a sampling frequency of $1 \mathrm{~Hz}$.

\section{Data Processing}

The obtained data were digitally low-pass filtered and decimated to the sampling frequency of $0.0167 \mathrm{~Hz}$; the Fourier spectra of the time series were computed without removing the linear trend. Decimated time series, with a sampling frequency of $0.00083 \mathrm{~Hz}$, were used for studying drifts in the data. The linear trend in the time domain data was estimated by the least-squares fitting for the time interval from 21 March after the second channel potential was stabilized.

\section{Results}

The obtained amplitude spectra are compared in Fig. 2. All the spectra show a decrease in amplitude with an increasing frequency except the local maximums and mini-

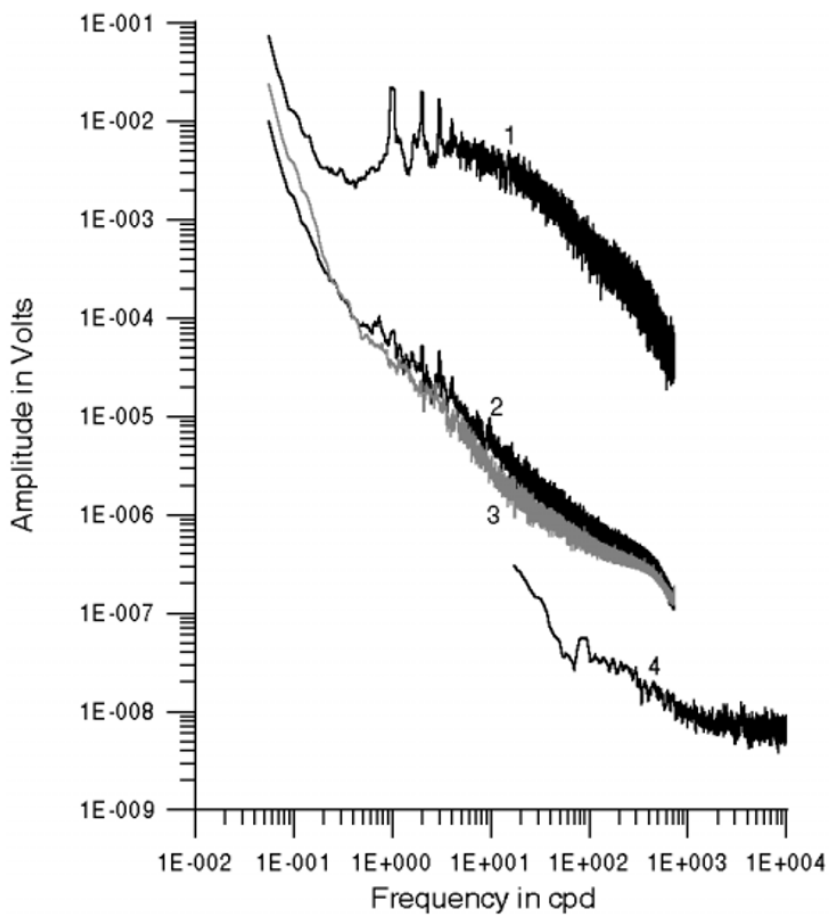

Fig. 2. The amplitude spectra of the JASC cable voltage (1) and the near-shore electrode potential difference for the first (2) and the second (3) pairs. The fourth curve (4) is the ADC noise spectrum.

mums seen for channel 1. The level of the JASC cable voltage spectrum is about three orders of magnitude larger than those of the other two channels for frequencies above $1 \mathrm{cpd}$. There is a small amplitude discrepancy between the spectra of channels 2 and 3. Both spectra levels are larger than that of the ADC noise spectrum, which was estimated by shorting the channel input and recording the output noise. The signals of channels 2 and 3 are larger than the ADC noise by about one order of magnitude, hence they are thought to be a result of electrode instabilities, since signals induced in the supplying wires by magnetic field variations are negligible in this frequency range. At the lowest frequencies, all spectra merge closely within one order of magnitude, which indicates a low $\mathrm{S} / \mathrm{N}$ ratio in this frequency range. It is worthwhile to note that the solar daily harmonics are present in the first and second channels, while they are absent in the third one. The coherence plots for the multiple coherence between the JASC cable voltage and voltage variations at channels 2 and 3 and the ordinary coherence function between channels 2 and 3 are presented in Fig. 3 . Both have high coherence at the lowest frequencies, indicating the presence of coherent low-frequency noise in the potentials of the titanium electrodes at the Nakhodka station. The solar daily harmonics are evident in the multiple coherence function and are strongly depressed in ordinary function in accordance with the spectra's structure. It is noticeable that the increased values of the ordinary coherence function appeared at frequencies of nearly $50 \mathrm{cpd}$ and lower. The linear trends in the time domain in channels 2 and 3 data are presented in Fig. 4. The onset of the plot is the ninth day from the beginning of the experiment, when the channel 2 electrode-pair potential was stabilized. Yet, 


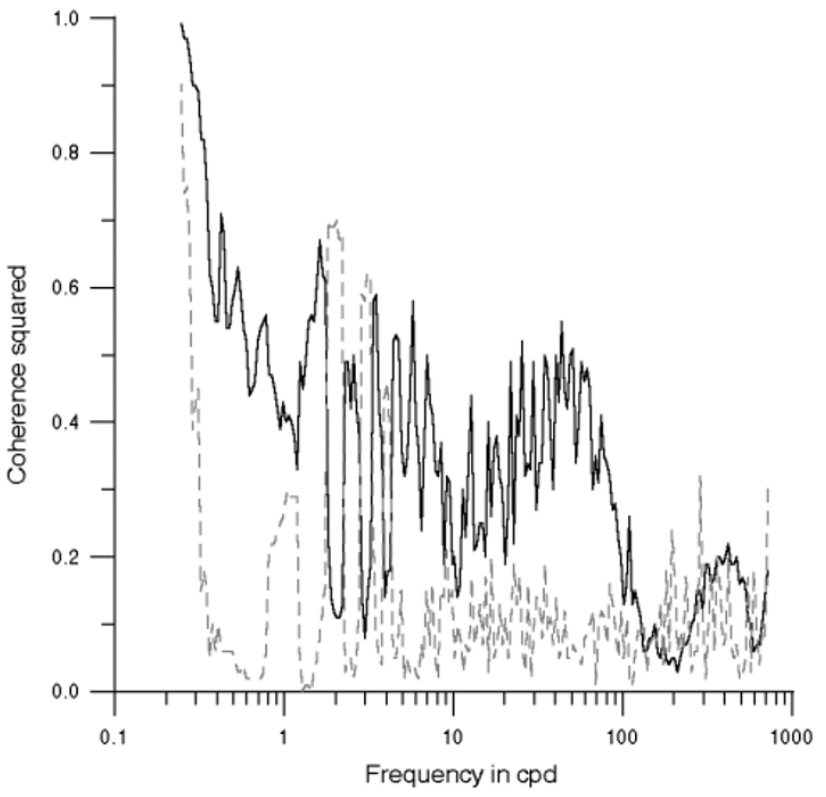

Fig. 3. The coherence plots for the multiple coherence between JASC data and channels 2 and 3 (grey broken line) and ordinary coherence between channels 2 and 3 (solid line).

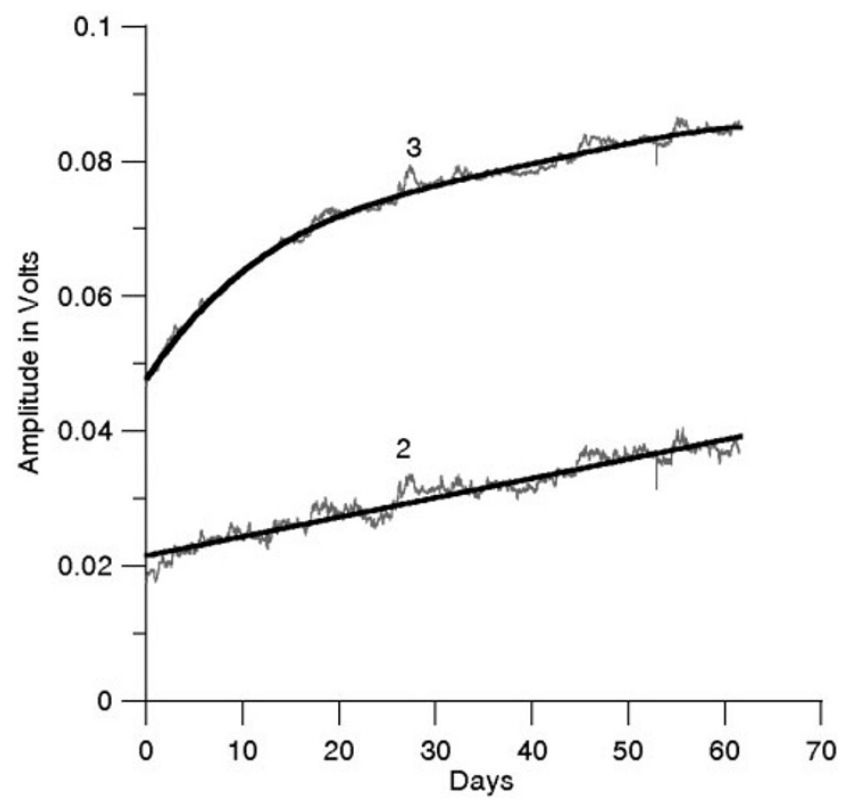

Fig. 4. Temporal changes in electrode voltage for channels 2 and 3 . Thick lines for channels 2 and 3 are linear and fourth polynomial, respectively. Both data are well approximated by linear trends after $\sim 30$ days.

this time interval was insufficient for the channel 3 electrode pair to be stabilized. Nevertheless, in the right part of the plot, linear trends are evident and both trends are parallel. The estimated linear drift coefficient is $0.28 \pm 0.0011$ $\mathrm{mV} /$ day. The low error is due to the absence of ionospheric signals in this case. For the same time interval, the estimated linear drifts in the JASC data and in the first channel are $0.34 \pm 0.146 \mathrm{mV} /$ day and $0.33 \pm 0.145 \mathrm{mV} /$ day, respectively. The large ionospheric signals in these channels are the reason of increasing errors in the estimated linear trend

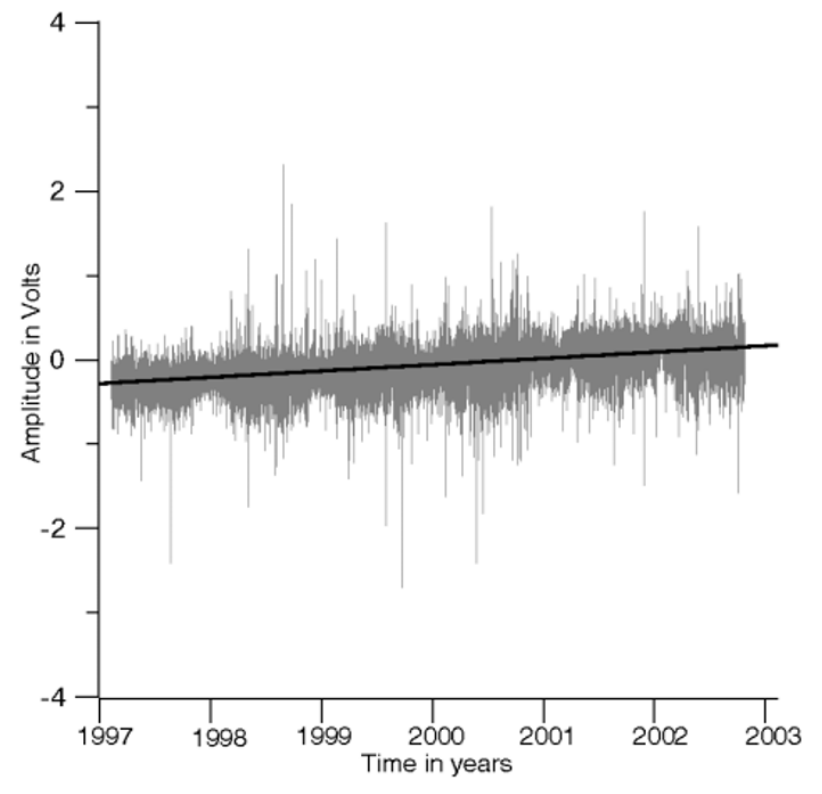

Fig. 5. The least-square fitting of the linear trend in the JASC data at a time interval of 6 years.

coefficients.

\section{Discussion}

The estimated linear drift in the electrode-pair voltage is near to that estimated from the JASC cable data, 0.21 $\mathrm{mV} /$ day $\left(=3.0 \times 10^{-4} \mathrm{mV} / \mathrm{km} \cdot\right.$ day $\left.\times 700 \mathrm{~km}\right)$ reported by Shimizu et al. (1998) for the time interval from 1 February until 1 December 1997. Our estimation of the linear trend in the JASC data for the time interval from 7 February 1997 until 21 October 2002 gives the value $0.20 \pm 0.001 \mathrm{mV} /$ day $\left(=2.9 \times 10^{-4} \mathrm{mV} / \mathrm{km} \cdot\right.$ day $\left.\times 700 \mathrm{~km}\right)($ see Fig. 5). This close agreement confirms the high stability of the JASC cable linear-trend coefficient. As was mentioned above, during our experiment in 2003, the linear drifts in the JASC and ADC E-24 first channel data were higher, $0.34 \pm 0.146$ $\mathrm{mV} /$ day and $0.33 \pm 0.145 \mathrm{mV} /$ day, respectively. Comparing the latter with the electrode pair linear drift, taking into account the error levels, we conclude that the electrode voltage variation is the main reason for the trend in the JASC and the first channel data during the experiment. Although Perrier et al. (1997) observed a linear type of drifts for unpolarized sea electrodes in the laboratory during a year-long experiment, the coefficient is substantially smaller than the present data. The dependence of the titanium electrode potential upon the temperature, pressure and sea-water salinity, etc., has not been well studied. In our experiment, a linear drift of $\sim 0.28 \pm 0.0011 \mathrm{mV} /$ day, which is comparable to the above JASC linear trend of $\sim 0.20 \pm 0.001 \mathrm{mV} /$ day, is observed over a period from one to two months after the disconnection of the electrodes. Although this drift rate may be reduced over a much longer elapsed time, the present result is in favor of the conclusion that the linear trend in the JASC cable data is partially caused by electrode noise. The same difference in these linear drifts is presumably caused by the remote electrode near the Japanese coast. At a sea depth of $1.5 \mathrm{~km}$, the electrode potential is influenced by different 
sea-water dynamics, high pressure, low temperature and the different degree of sea-water salinity. Therefore, to study the Earth's electrical currents of internal origin (Meloni et al., 1983; Shimizu et al., 1998; Shimizu and Utada, 2003), we need to reduce or remove the electrode noise from the JASC cable data on the basis of special efforts to control the noise parameters. It is desirable to simultaneously, monitor the electrode potential variation e.g. by measuring the potential differences between closely spaced multiple electrodes, including the electrode used for the JASC cable observation. This might enable us to estimate the time dependence of the electrode voltage drift and to apply some procedures to remove it.

Acknowledgments. We are greatly indebted to the staff of the Nakhodka telecommunication station for helpful cooperation and provisions for the experiment, and to Dr. R. G. Kulinich for aid in providing research facilities. We would like to thank Prof. H. Utada for giving much attention to the JASC cable investigation. Comments from referees were greatly appreciated. This work was supported by RFBR Grant 04-05-64233.

\section{References}

Chave, A. D., D. S. Luther, and L. V. Medford, Geoelectric field measurement on a planetary scale: oceanographic and geophysical applications, Geophys. Res. Lett., 19, 1411-1414, 1992.

Constable, S. C., A. S. Orange, G. M. Hoversten, and H. F. Morrison, Marine magnetotellurics for petroleum exploration part 1: A sea-floor equipment system, Geophysics, 63, 816-825, 1998.

Egbert, G. D., J. R. Booker, and A. Schultz, Very long period magnetotellurics at tucson observatory: estimation of impedances, J. Geophys. Res., 97, 15113-15128, 1992.
Lanzerotti, L. J., D. J. Thomson, A. Meloni, L. V. Medford, and C. G. Maclennan, Electromagnetic study of Atlantic continental margin using a section of a transatlantic cable, J. Geophys. Res., 91, 7417-7427, 1986.

Lorne, B., F. Perrier, and J. P. Avouac, Streaming potential measurements 1. Properties of the electrical double layer from crushed rock samples, J. Geophys. Res., 104, 17857-17877, 1999.

Medford, L. V., L. J. Lanzerotti, J. S. Kraus, and C. G. Maclennan, Transatlantic Earth potential variations during the March 1989 magnetic storm, Geophys. Res. Lett., 16, 1145-1148, 1989.

Meloni, A., L. J. Lanzerotti, and G. P. Gregori, Induction of currents in long submarine cables by natural phenomena, Rev. Geophys. Space Physics, 21, 795-803, 1983.

Perrier, F. E., G. Petiau, G. Clerc, V. Bogorodsky et al., A one-year systematic studies of electrodes for long period measurements of the electric field in geophysical environments, J. Geomag. Geolectr., 49, 16771696, 1997.

Petiau, G. and A. Dupis, Noise, temperature coefficient, and long time stability of electrodes for telluric observations, Geoph. Prosp., 28, 792804, 1980.

Shimizu, H. and H. Utada, Detectability of decadal variations of the surface electric potential generated by zonal oscillating flows in Earth's core, in The $3^{\text {rd }}$ International Workshop on Scientific Use of Submarine Cable and Related Technologies, 315 pp., IEEE, Tokyo, 2003.

Shimizu, H., T. Koyama, and H. Utada, An observational constraint on the strength of the toroidal magnetic field at the CMB by time variation of submarine cable voltages, Geophys. Res. Lett., 25, 4023-4026, 1998.

Vanyan L. L., H. Utada, H. Shimizu, Y. Tanaka et al., Studies on the lithosphere and the water transport by using the Japan Sea submarine cable (JASC): 1. Theoretical considerations, Earth Planets Space, 50, 35-42, 1998.

Webb, S. C., S. C. Constable, C. S. Cox, and T. K. Deaton, A sea electric field instrument, J. Geomag. Geolectr., 37, 1115-1129, 1985.

S. S. Starjinsky (e-mail: starjinsky_ss@yahoo.com) and V. M. Nikiforov 